\title{
BMJ Open Addition of arterial spin-labelled MR perfusion to conventional brain MRI: clinical experience in a retrospective cohort study
}

\author{
Puneet Belani, ${ }^{1}$ Shingo Kihira (D) , ${ }^{1}$ Felipe Pacheco, ${ }^{1}$ Puneet Pawha, ${ }^{1}$ \\ Giuseppe Cruciata, ${ }^{1}$ Kambiz Nael $^{2}$
}

To cite: Belani P, Kihira S, Pacheco F, et al. Addition of arterial spin-labelled MR perfusion to conventional brain MRI: clinical experience in a retrospective cohort study. BMJ Open 2020;10:e036785. doi:10.1136/ bmjopen-2020-036785

- Prepublication history for this paper is available online. To view these files, please visit the journal online (http://dx.doi org/10.1136/bmjopen-2020036785).

Received 06 January 2020 Revised 21 April 2020 Accepted 06 May 2020

\section{A) Check for updates}

\section{(c) Author(s) (or their} employer(s)) 2020. Re-use permitted under CC BY-NC. No commercial re-use. See rights and permissions. Published by BMJ.

${ }^{1}$ Department of Radiology, Icahn School of Medicine at Mount Sinai, New York, New York, USA ${ }^{2}$ Department of Radiological Sciences, David Geffen School of Medicine, University of California Los Angeles, Los Angeles, California, USA

Correspondence to Dr Kambiz Nael, kambiznael@gmail.com

\section{ABSTRACT}

Objective The usage of arterial spin labelling (ASL) perfusion has exponentially increased due to improved and faster acquisition time and ease of postprocessing. We aimed to report potential additional findings obtained by adding ASL to routine unenhanced brain MRI for patients being scanned in a hospital setting for various neurological indications.

Design Retrospective.

Setting Large tertiary hospital.

Participants 676 patients.

Primary outcome Additional findings from ASL sequence compared with conventional MRI.

Results Our patient cohorts consisted of 676 patients with 257 with acute infarcts and 419 without an infarct. Additional findings from ASL were observed in 13.9\% (94/676) of patients. In the non-infarct group, additional findings from ASL were observed in 7.4\% (31/419) of patients, whereas in patients with an acute infarct, supplemental information was obtained in $24.5 \%(63 / 257)$ of patients.

Conclusion The addition of an ASL sequence to routine brain MRI in a hospital setting provides additional findings compared with conventional brain MRI in about $7.4 \%$ of patients with additional supplementary information in $24.5 \%$ of patients with acute infarct.

\section{INTRODUCTION}

Neuroimaging constitutes a significant portion of hospital-based imaging both for CT and MRI. ${ }^{1-3}$ Although most acute hospital-based neurological encounters can be assessed with CT, MRI offers an attractive alternative due to the lack of ionising radiation and superior soft tissue contrast that is extremely useful for a detailed evaluation of brain tissue. In fact, the Appropriateness Criteria of the American College of Radiology list MRI as the desired imaging modality for most neurological symptoms, including headache, focal neurological deficits, altered mental status, ataxia, seizure and vision loss. ${ }^{4}$ A conventional brain MRI consists of several weightings and is often constructed
Strengths and limitations of this study

To our knowledge, this is the first study to assess additional findings from arterial spin labelling (ASL) MRI compared with conventional MRI for a variety of neurological pathology seen in routine clinical practice.

- The study consists of a relatively large patient cohort, which strengthens the validity of the study.

- There was only one observer in the study, which limits the assessment of reproducibility.

- This is a retrospective cohort study, which limits the determination of the effect of ASL images on clinical management.

- The 1.5 $\mathrm{T}$ is not a preferred magnetic field for ASL, which may suggest that additional findings may be underestimated in this study.

with at least five sequences including T1, T2, Fluid-attenuted inversion recovery (FLAIR), gradient-recalled-echo (GRE) and diffusionweighted imaging (DWI). ${ }^{56}$

Arterial spin labelling (ASL) is a contrastfree MR perfusion technique that uses magnetically labelled water as a freely diffusible tracer to measure cerebral blood flow (CBF). ${ }^{7-9}$ Twenty-fiveyears after its introduction and with significant advances in sequence design, hardware technology and postprocessing techniques, ASL has become readily available for routine clinical practice in recent years. ${ }^{10}{ }^{11}$ In particular, ASL has shown promising results in the assessment of acute neurological disorders such as stroke, ${ }^{12-16}$ transient ischaemic attack (TIA), ${ }^{17} 18$ seizure, ${ }^{19}{ }^{20}$ migraine headaches, ${ }^{21}$ as well as various neuropsychiatric diseases such as major depressive disorder, ${ }^{22} 23$ Alzheimer's dementia ${ }^{24-26}$ and Parkinson's disease. ${ }^{27-30}$ In this study, we aimed to investigate additional findings from ASL imaging 
compared with routine unenhanced brain MRI scan in a hospital setting.

\section{MATERIALS AND METHODS Study population}

This is a single-centre retrospective study. Patients who presented to our inpatient and emergency department from January 2015 to September 2018 were included if they had concurrent ASL imaging obtained with a routine unenhanced brain MRI scan. A total of 5676 brain MRIs were performed in our hospital (inpatient and emergency department) of which 700 patients had MRI with concurrent ASL imaging that met our inclusion and exclusion criteria. Contrast-enhanced brain MRIs did not have ASL and therefore were not included in this study. Indications for these studies included a variety of neurological indications seen in routine clinical settings such as headaches, altered mental status, neurological deficits and seizures. Specific protocols, for example, epilepsy protocol, multiple-sclerosis protocol, internal auditory canal protocol and brain tumour protocol, did not have ASL imaging routinely included and were excluded from the study. Paediatric patients were excluded. Patients were excluded if the ASL images were severely limited by motion artefact or insufficient tagging. This yielded a total of 700 cases with concurrent ASL imaging. Of which, 24 were excluded due to various reasons: motion artefact and insufficient tagging $(n=14)$, inability of the patient to complete the ASL sequence $(n=6)$ and susceptibility artefacts $(n=4)$. This resulted in the inclusion of a total of 676 patients in our study.

\section{Image acquisition}

Imaging protocol for brain MRI included the acquisition of sagittal T1, axial T2, axial GRE, axial FLAIR, axial DWI and axial ASL. Image acquisition was performed using two $1.5 \mathrm{~T}$ clinical MRI units (GE Optima MR450w; GE Medical Systems, Milwaukee) with an eight-channel head coil for signal reception. Three-dimensional (3D) pseudocontinuous ASL with a fast spin-echo stack-ofspiral readout with eight interleaves was used with each spiral arm including 512 sampling points. The following parameters were applied: repetition time (TR)/echo time (TE) : 4525/11 ms; field-of-view (FOV): $24 \times 24 \mathrm{~cm}$, matrix size: $64 \times 64 \mathrm{~mm}, 30$ slices each $4 \mathrm{~mm}$ thick; and number of excitation $(\mathrm{NEX})=3$. Pseudocontinuous spin labelling was performed for $1.5 \mathrm{~s}$ before postlabelling delay of $2 \mathrm{~s}$. Background suppression was achieved by applying inversion pulses on the volume to be imaged, allowing for the increase in the sharpness of the bolus. ${ }^{31}$ This setting resulted in the acquisition of a $3 \mathrm{D}$ voxel size of $3.8 \times 3.8$ $\times 4 \mathrm{~mm}^{3}$ during 4:30 min. Scanner software was used for online image reconstruction. Pairwise subtraction between label and control images was obtained and averaged to generate the mean difference and converted to CBF maps based on a previously published model. ${ }^{10}$ After automated reconstruction by the scanner software, digital imaging and communications in medicine (DICOM) CBF maps along with all other anatomical imaging were sent to picture archiving and communication system (PACS) and were available for interpretation.

\section{Image analysis}

For the purpose of this study, ASL images were retrospectively evaluated by one board-certified neuroradiologist for the detection of regional hypoperfusion or hyperperfusion. The observer was blinded to the final diagnosis to avoid bias. 'Additional diagnostic findings' were noted in the context of conventional images and final diagnosis. For example, in a patient with a diagnosis of TIA, for additional findings to be counted, initial ASL analysis (blinded to TIA diagnosis) must have been recorded as regional hypoperfusion. In a review of conventional imaging for this case, there should be no evidence of acute infarction. Image analysis was performed on a PACS (Centricity, GE Healthcare, Chicago, Illinois, USA) station without any additional software. Additional findings were noted if the ASL finding (1) suggested a new diagnosis, (2) helped narrow the differential or (3) prompted further imaging/workup, which would not have been considered with conventional imaging only.

In patients with acute infarct, the diagnosis was made by DWI and therefore ASL findings if any were considered supplementary and not as an 'additional finding'. In patients who had multiple regions of perfusion abnormality or a mixture of hypoperfusion and hyperperfusion, the most acutely relevant ASL finding was regarded as the predominant perfusion pattern. For example, in a patient with an acute infarct with associated luxury hyperperfusion and a chronic infarct elsewhere with corresponding hypoperfusion, the hyperperfusion related to acute infarction was included in the data analysis.

Final diagnoses were extracted from patients' discharge summary and patients were categorised into two groups: acute infarction and non-infarction. Percentages of ASL abnormalities were calculated and subdivided in each category.

\section{Patient and public involvement}

Patients and/or the public were not involved in the design, or conduct, or reporting, or dissemination plans of this research.

\section{RESULTS}

\section{Patient cohort}

Our final cohort included 676 patients. The mean \pm SD of age (years) was $62 \pm 18$ with a median of 64 . Among our cohort, 312 were men and 364 were women. CBF analysis of the cohort revealed 79 patients with hyperperfusion, 250 with hypoperfusion and 347 with normal perfusion.

\section{Group with acute ischaemic infarction}

A total of 257 of $676(38.0 \%)$ patients had acute infarcts (109 with small vessel infarcts and 148 with large vessel 


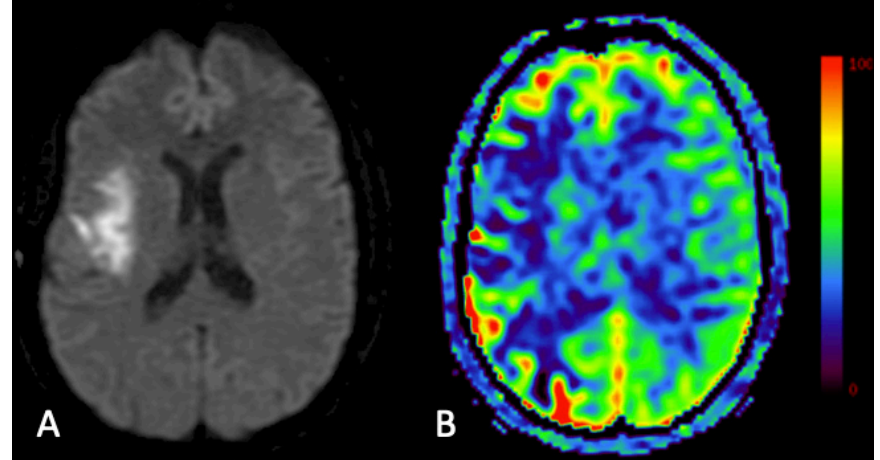

Figure 1 Patient presented to the emergency department with sudden left facial and arm weakness and underwent a standard stroke protocol with non-contrast brain MRI. $\mathrm{DWI}(\mathrm{A})$ and $\mathrm{ASL}-\mathrm{CBF}(\mathrm{B})$ are shown. There is restricted diffusion in the right middle cerebral artery (MCA) territory involving the anterior and posterior portion of right insula. ASL demonstrated corresponding and larger hypoperfusion around the infarction bed suggesting persistent hypoperfusion of ischaemic territory ('penumbra'). ASL, arterial spin labelling; CBF, cerebral blood flow; DWI, diffusion-weighted imaging.

infarcts). ASL provided supplementary information in 3 of $109(2.8 \%)$ patients with small vessel infarcts and 60 of $148(40.5 \%)$ patients with large vessel infarcts. Among 63 patients with acute infarct in whom ASL showed supplementary information, 47 patients had luxury perfusion of infarction bed representing infarct reperfusion (figure 1) and 16 patients had corresponding and larger hypoperfusion around the infarction bed suggesting persistent hypoperfusion of ischaemic territory (figure 2). ASL's additional findings in these patients are summarised in table 1.

\section{Group without acute infarction}

Out of 419 patients who did not have an acute ischaemic infarct, additional findings were observed on ASL in 31

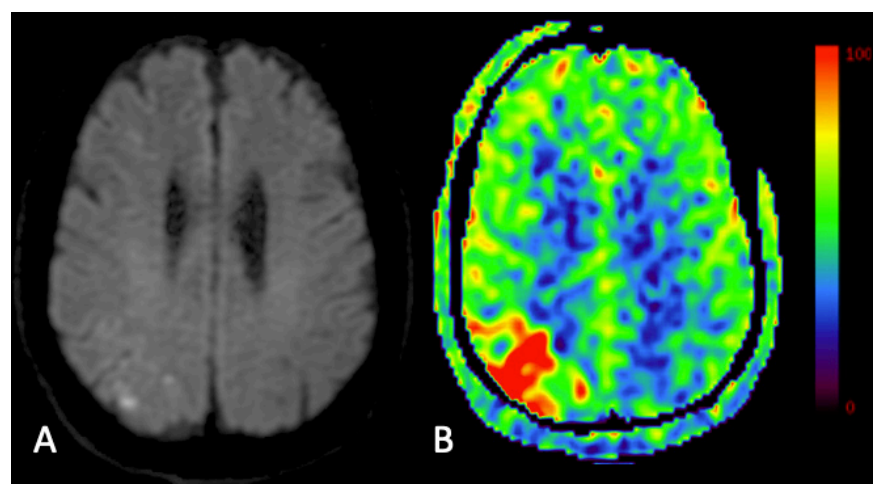

Figure 2 Patient presented to the emergency department with altered mental status. DWI (A) and ASL-CBF (B) are shown. There are several foci of restricted diffusion in the right middle cerebral artery (MCA) / posterior cerebral artery (PCA) border zone. ASL demonstrated corresponding hyperperfusion around the infarction bed representing luxury perfusion. ASL, arterial spin labelling; CBF, cerebral blood flow; DWI, diffusion-weighted imaging.
Table 1 Supplementary information provided by ASL in patients with acute infarction

\begin{tabular}{llll}
\hline Category & $\begin{array}{l}\text { No of } \\
\text { patients }\end{array}$ & $\begin{array}{l}\text { Additional } \\
\text { findings }\end{array}$ & $\begin{array}{l}\text { Yield of ASL } \\
(\%)\end{array}$ \\
\hline Acute infarct & $257(52 / 148)$ & $63(47 / 16)$ & 24.51 \\
$\begin{array}{l}\text { Small vessel } \\
\text { infarct }\end{array}$ & $109(0 / 109)$ & $3(0 / 3)$ & 2.80 \\
$\begin{array}{l}\text { Large vessel } \\
\text { infarct }\end{array}$ & $148(52 / 96)$ & $60(47 / 13)$ & 40.50 \\
\hline
\end{tabular}

Data presented within parenthesis represent the number of hyperperfusion and number of hypoperfusion on ASL. ASL, arterial spin labelling.

patients (7.4\%) (table 2). In these cases, ASL helped diagnose certain stroke mimics that otherwise would not have been as conspicuous or easily diagnosed on conventional MR sequences. Hypoperfusion on ASL was observed in patients with TIA or unsuspected vascular stenosis $(\mathrm{n}=12)$ (figure 3). Hyperperfusion was seen for infection/ encephalitis $(n=7)$, postictal state $(n=4)$ (figure 4$)$, hypervascular masses $(\mathrm{n}=4)$ (figure 5), posterior reversible encephalopathy syndrome $(n=2)$, migraine $(n=1)$ and Wernicke encephalopathy $(n=1)$.

\section{DISCUSSION}

Due to its non-invasive nature and ability to measure cerebral perfusion, ASL can provide important physiological information that would not be available through conventional MRI. With recent technical advances including fast imaging tools and automated postprocessing, ASL can be performed routinely in clinical practice with minimal burden on acquisition time or radiologists' workflow while providing valuable physiological information.

In this study, we showed that ASL can be added successfully to routine unenhanced brain MRI protocol in a hospital setting and provide valuable diagnostic and

Table 2 Additional diagnostic findings from ASL for patients in the non-infarct cohort

\begin{tabular}{llll} 
& $\begin{array}{l}\text { Patients } \\
\text { (n) }\end{array}$ & $\begin{array}{l}\text { Additional } \\
\text { findings }\end{array}$ & $\begin{array}{l}\text { Yield of } \\
\text { ASL (\%) }\end{array}$ \\
\hline Non-infarct/Other & 419 & 31 & 7.40 \\
TIA/vascular stenosis & & 12 & \\
Infection & 7 & \\
Seizure & 4 & \\
Tumour/mass & 4 & \\
PRES & 2 & \\
Migraine & 1 & \\
Wernicke & 1 & \\
\hline
\end{tabular}

ASL, arterial spin labelling; PRES, posterior reversible encephalopathy syndrome; TIA, transient ischaemic attack. 


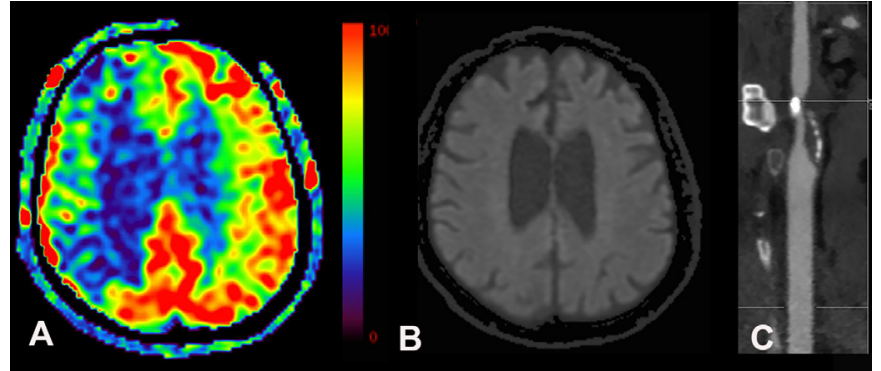

Figure 3 Patient presented with recurrent episodes of syncope and unresponsiveness. ASL-CBF (A) showing significantly decreased perfusion in the right hemisphere along the right anterior cerebral artery (ACA) and middle cerebral artery (MCA) distributions suggesting underlying carotid stenosis. Axial DWI (B) showing no acute infarction. Based on these findings, a CT angiogram of the head and neck was recommended and performed, which revealed approximately $93 \%$ stenosis at the right internal carotid artery (ICA) origin (C) due to mixed atherosclerotic plaque. The patient was diagnosed with symptomatic high-grade carotid stenosis and was subsequently scheduled for carotid endarterectomy. ASL, arterial spin labelling; CBF, cerebral blood flow; DWI, diffusion-weighted imaging.

prognostic findings that would not be available through conventional MRI. In particular, we highlight two findings.

First, in a subset of patients without acute infarction, ASL provided additional diagnostic findings in $31(7.4 \%)$ patients. These included patients with diagnoses of seizure, migraine, central nervous system (CNS) infection, haemodynamic impairment, such as TIA or underlying vascular stenosis, or hypervascular mass lesions.

Migraine and seizure (postictal) both have been reported as common stroke mimics. ${ }^{32-34}$ Identifying a mimic from a stroke is critical in regard to the financial burden and avoiding the subsequent unnecessary medical management of these patients. These include direct and indirect hospital costs of occupying a stroke bed and tissue plasminogen activator (tPA) administration, not

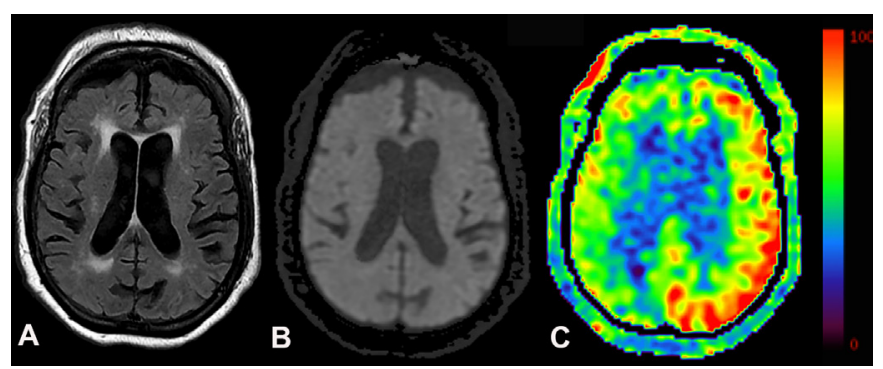

Figure 4 Patient presented to the emergency department with altered mental status and unexplained loss of consciousness and underwent a routine non-contrast brain MRI. Axial FLAIR (A), DWI (B) and ASL-CBF (C) are shown. There is regional hyperperfusion involving the left cerebral hemisphere without associated FLAIR hyperintensity or restricted diffusion. Seizure and postictal status were correctly suggested based on increased non-territorial hyperperfusion. ASL, arterial spin labelling; CBF, cerebral blood flow; DWI, diffusion-weighted imaging.

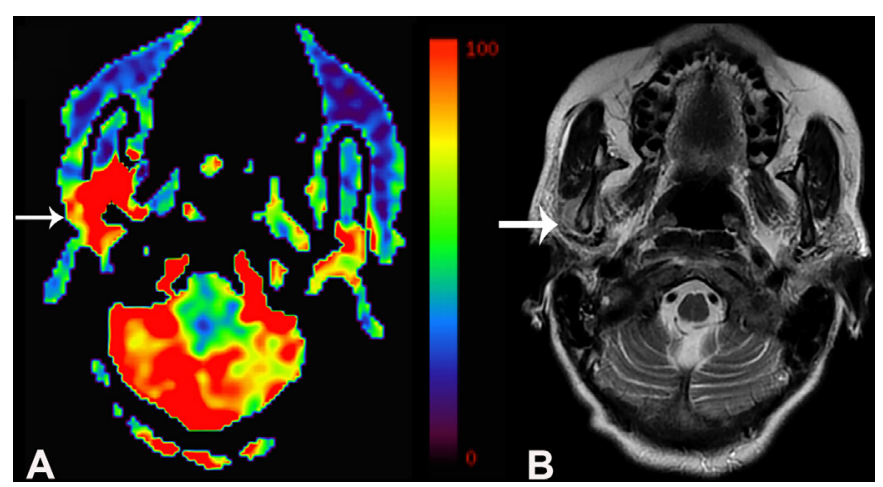

Figure 5 Patient presented with a severe headache and underwent routine brain $\mathrm{MRI}$ in the emergency department. Axial ASL-CBF (A) showing hyperperfusion in the right masticator space and around the mandibular condyle (arrow). This led to a closer inspection of the other sequences and the axial T2 image (B), showing thickening of the right temporomandibular joint capsule and abnormal signal in the mandibular condyle (arrow). Further workup led to the diagnosis of multiple myeloma. ASL, arterial spin labelling; CBF, cerebral blood flow.

to mention the financial burden on the patient after discharge such as the effect on driving and medical insurance premiums that are often not considered. In our study, we showed that non-territorial hyperperfusion on ASL-CBF is helpful in the correct diagnosis of patients with migraine and seizure in the postictal state (figure 4) as has been reported previously. ${ }^{19} 21$ Prior ASL studies in postictal states have shown hyperperfusion in the epileptogenic grey matter surrounding parenchyma in a non-vascular territory. ${ }^{35}$ There were four cases in our study where additional findings were observed on ASL compared with conventional MRI.

Vascular disorders were the most common type of pathology where additional diagnostic findings were observed from ASL images in the non-infarct group. The most common of these were in identifying territorial hypoperfusion with areas of stenosis that were or were not known but identified on MRI based on locally decreased CBF along a vascular territory (figure 3). Perfusion deficit has also been also reported in patients with TIA and in fact up to $30 \%$ of patients with DWI-negative TIA can have regional perfusion abnormalities. ${ }^{36-38} \mathrm{We}$ correctly identified seven patients with TIA in our cohort as having territorial hypoperfusion. One last case among the vascular disorder group was a patient with a very small dural arteriovenous (AV) fistula identified by hyperperfusion on ASL, which otherwise would have been a more challenging diagnosis to make on routine MRI. The usage of ASL for dural AV fistula has also been described in the prior literature. $^{39}$

CNS infection, such as leptomeningitis or encephalitis, was another subgroup of patients in whom additional diagnostic findings were observed on ASL imaging compared with conventional MRI. The diagnosis was suggested based on the areas of leptomeningeal FLAIR hyperintensity with associated elevated CBF. Typically, the 
history in these patients was non-specific such as headache and not all patients presented with high fever or nuchal rigidity. Observing subtle sulcal FLAIR signal abnormality particularly in the occipital lobes is not uncommon in a high-volume inpatient setting. The clinical significance of this finding is not always easily determined, especially in patients with an ambiguous clinical presentation. When such a finding is observed in conjunction with elevated $\mathrm{CBF}$, the interpreting radiologist may suggest further workup with lumbar puncture and cerebrospinal fluid analysis to confirm the diagnosis of meningitis. ${ }^{40}$ In one case, ASL also helped diagnose hyperperfusion secondary to phlegmonous changes in the parapharyngeal space in a patient with recent neck surgery. The last group of patients for whom we found additional diagnostic findings from ASL was for hypervascular mass lesions $(n=4)$ that otherwise would have been difficult to identify on an unenhanced MRI (figure 5).

The second finding we would like to highlight in this study is that in a subset of patients with acute infarct, ASL provided supplementary information to conventional MRI with potential prognostic or therapeutic implications. The authors decided to categorise these patients separately as diagnosis of infarction is made with high confidence by DWI images and ASL information will have a negligible value from additional findings. However, ASL can provide supplementary physiological information about the infarction bed with prognostic and therapeutic value. Having ASL hypoperfusion associated with the ischaemic territory in patients with acute infarction has been reported to worsen functional outcome and infarct growth. ${ }^{11}$ The so-called DWI-ASL mismatch, where ASL defect is larger than a DWI lesion (figure 1), has been used for clinical decision making in prior studies, ${ }^{38} 41$ however, this should be interpreted with caution as ASL can notoriously overestimate the extent of hypoperfusion due to problem associated with sluggish blood flow and arterial transit delay. ${ }^{42}{ }^{43}$ Patients with subjectively large areas of mismatch were interpreted as having hypoperfusion around a core infarct, which may guide the treating physician in medical management.

Having ASL hyperperfusion associated with ischaemic bed in patients with acute ischaemic stroke can be valuable (figure 2), especially with large vessel involvement. ${ }^{11}$ Prior studies have shown that hyperperfusion on ASL within or around DWI lesions is associated with hypertension $^{16}{ }^{44}$ or developing parenchymal haemorrhage. ${ }^{16}$ Thus, identification of hyperperfusion may encourage clinicians to ensure closer monitoring and tighter blood pressure regulation to avoid complications such as parenchymal haemorrhage. In contrast, it has been shown that focal hyperperfusion early after thrombolysis is associated with a smaller final infarct volume and with the improved functional outcome at 24 hours and 3 months even despite having a higher chance of parenchymal haemorrhage. ${ }^{454}$ It should be emphasised that some of this presumed hyperperfusion may indeed represent arterial transit delay related to sluggish flow and collateral vessels in particular considering applied $1.5 \mathrm{~T}$ imaging in our study. ${ }^{17}$

Our study has several limitations. First, this was a retrospective cohort study, which limited the determination of the effect of ASL images on clinical management. A prospective study would have allowed us to assess for clinical implications and outcomes based on these additional findings from ASL imaging. Second, these additional findings were inherently based on the diagnostic interpretations of the conventional MR sequences and may be operator dependent. For example, depending on the experience of the radiologist, the interpretation from conventional sequences may differ, which would affect the determination of what was considered an additional finding on ASL imaging. Additionally, we acknowledge that the patient population in our study is not representative of the general population as our cohort consisted of patients from inpatient and emergency settings and additional findings on ASL may vary in a different clinical scenario. Lastly, we acknowledge that ASL image acquisition at $1.5 \mathrm{~T}$ in our study is suboptimal as ASL is best optimised for $3 \mathrm{~T}$ MR scanners. Although this is a limitation, it is plausible that the additional findings from ASL may have been underestimated by our study.

In conclusion, the addition of an ASL sequence to routine brain MRI provides additional diagnostic findings compared with conventional brain MRI in about $7.4 \%$ of patients with additional supplementary information in $24.5 \%$ of patients with acute infarct. The additional findings observed on ASL may aid in the differential diagnosis of neurological processes such as TIA, important stroke mimics, such as seizure and migraine, and hypervascular lesions.

Contributors Guarantor of integrity of the entire study: PB, SK, PP and KN. Study concepts and design: PB, PP and KN. Literature research: PB, SK, FP, PP, GC and KN Clinical studies: PB, FP, PP, GC and KN. Experimental studies/data analysis: PB, SK, FP and KN. Statistical analysis: PB, SK and KN. Manuscript preparation: PB, SK, PP and KN. Manuscript editing: PB, SK, PP and KN.

Funding The authors have not declared a specific grant for this research from any funding agency in the public, commercial or not-for-profit sectors.

Competing interests None declared.

Patient consent for publication Not required.

Ethics approval This study is approved by the Mount Sinai Hospital Institutional Review Board with a waiver of informed consent (IRB\# MS312-789).

Provenance and peer review Not commissioned; externally peer reviewed.

Data availability statement All data relevant to the study are included in the article or uploaded as supplementary information. All data are included in the manuscript. No additional data are available.

Open access This is an open access article distributed in accordance with the Creative Commons Attribution Non Commercial (CC BY-NC 4.0) license, which permits others to distribute, remix, adapt, build upon this work noncommercially, and license their derivative works on different terms, provided the original work is properly cited, appropriate credit is given, any changes made indicated, and the use is non-commercial. See: http://creativecommons.org/ licenses/by-nc/4.0/.

ORCID iD

Shingo Kihira http://orcid.org/0000-0003-1001-8741 


\section{REFERENCES}

1 Rankey D, Leach JL, Leach SD. Emergency MRI utilization trends at a tertiary care academic medical center: baseline data. Acad Radiol 2008;15:438-43.

2 Arasu VA, Abujudeh HH, Biddinger PD, et al. Diagnostic emergency imaging utilization at an academic trauma center from 1996 to 2012. J Am Coll Radiol 2015;12:467-74.

3 Ahn S, Kim WY, Lim KS, et al. Advanced radiology utilization in a tertiary care emergency department from 2001 to 2010. PLoS One 2014;9:e112650.

4 Subramaniam RM, Kurth DA, Waldrip CA, et al. American College of radiology appropriateness criteria: advancing evidence-based imaging practice. Semin Nucl Med 2019;49:161-5.

5 Ellingson BM, Bendszus M, Boxerman J, et al. Consensus recommendations for a standardized brain tumor imaging protocol in clinical trials. Neuro Oncol 2015;17:1188-98.

6 Edelstein WA, Mahesh M, Carrino JA. MRI: time is dose--and money and versatility. J Am Coll Radiol 2010;7:650-2.

7 Williams DS, Detre JA, Leigh JS, et al. Magnetic resonance imaging of perfusion using spin inversion of arterial water. Proc Natl Acad Sci U S A 1992;89:212-6.

8 Detre JA, Rao H, Wang DJJ, et al. Applications of arterial spin labeled MRI in the brain. J Magn Reson Imaging 2012;35:1026-37.

9 Wintermark M, Sesay M, Barbier E, et al. Comparative overview of brain perfusion imaging techniques. Stroke 2005;36:e83-99.

10 Alsop DC, Detre JA, Golay X, et al. Recommended implementation of arterial spin-labeled perfusion MRI for clinical applications: a consensus of the ISMRM perfusion Study Group and the European Consortium for ASL in dementia. Magn Reson Med 2015;73:102-16.

11 Verclytte S, Fisch O, Colas L, et al. Asl and susceptibility-weighted imaging contribution to the management of acute ischaemic stroke. Insights Imaging 2017;8:91-100.

12 Oura D, Kawabori M, Niiya Y, et al. The validity of the acute stroke assessment using rapid Pseudo-continuous arterial spin labeling (ASAP-ASL) method for acute thrombectomy. J Neurosurg Sci 2018. doi:10.23736/S0390-5616.18.04607-6

13 Thamm T, Zweynert S, Piper SK, et al. Diagnostic and prognostic benefit of arterial spin labeling in subacute stroke. Brain Behav : 2019.

14 Yoo R-E, Yun TJ, Rhim JH, et al. Bright vessel appearance on arterial spin labeling MRI for localizing arterial occlusion in acute ischemic stroke. Stroke : 2015;46:564-7.

15 Tada Y, Satomi J, Abe T, et al. Intra-Arterial signal on arterial spin labeling perfusion MRI to identify the presence of acute middle cerebral artery occlusion. Cerebrovasc Dis 2014;38:191-6.

16 Yu S, Liebeskind DS, Dua S, et al. Postischemic hyperperfusion on arterial spin labeled perfusion MRI is linked to hemorrhagic transformation in stroke. J Cereb Blood Flow Metab 2015;35:630-7.

17 Zaharchuk G. Arterial spin label imaging of acute ischemic stroke and transient ischemic attack. Neuroimaging Clin N Am 2011;21:285-301.

18 Havsteen I, Willer L, Ovesen C, et al. Significance of arterial spin labeling perfusion and susceptibility weighted imaging changes in patients with transient ischemic attack: a prospective cohort study. BMC Med Imaging : 2018;18:24.

19 Kim BS, Lee S-T, Yun TJ, et al. Capability of arterial spin labeling MR imaging in localizing seizure focus in clinical seizure activity. Eur $J$ Radiol 2016;85:1295-303.

20 Bansal V, Kumar S, Sharma S, et al. Usefulness of pulsed arterial spin labeling magnetic resonance imaging in new-onset seizure patients and its comparison with dynamic susceptibility contrast magnetic resonance imaging. J Neurosci Rural Pract 2017;8:569-74.

21 Corno S, Giani L, Laganà MM, et al. The brain effect of the migraine attack: an ASL MRI study of the cerebral perfusion during a migraine attack. Neurol Sci 2018;39:73-4.

22 Liao W, Wang Z, Zhang X, et al. Cerebral blood flow changes in remitted early- and late-onset depression patients. Oncotarget 2017;8:76214-22.

23 Ramasubbu R, Brown EC, Marcil LD, et al. Automatic classification of major depression disorder using arterial spin labeling MRI perfusion measurements. Psychiatry Clin Neurosci 2019;73:486-93.
24 Riederer I, Bohn KP, Preibisch C, et al. Alzheimer Disease and Mild Cognitive Impairment: Integrated Pulsed Arterial Spin-Labeling MR and ${ }^{18}$ F-FDG PET. Radiology 2018;288:198-206.

25 McDade E, Kim A, Hughes T, et al. Cerebral amyloid related alterations in neuronal metabolism and the contribution of multimodal measures of vascular function. Neurology 2014.

26 Roquet D, Sourty M, Botzung A, et al. Brain perfusion in dementia with Lewy bodies and Alzheimer's disease: an arterial spin labeling MRI study on prodromal and mild dementia stages. Alzheimers Res Ther 2016;8.

27 Le Heron CJ, Wright SL, Melzer TR, et al. Comparing cerebral perfusion in Alzheimer's disease and Parkinson's disease dementia: an ASL-MRI study. J Cereb Blood Flow Metab 2014;34:964-70.

28 Melzer TR, Watts R, MacAskill MR, et al. Arterial spin labelling reveals an abnormal cerebral perfusion pattern in Parkinson's disease. Brain 2011;134:845-55.

29 Barzgari A, Sojkova J, Maritza Dowling N, et al. Arterial spin labeling reveals relationships between resting cerebral perfusion and motor learning in Parkinson's disease. Brain Imaging Behav 2019;13:577-87.

30 Wei X, Yan R, Chen Z, et al. Combined diffusion tensor imaging and arterial spin labeling as markers of early Parkinson's disease. Sci Rep 2016.

31 Mani S, Pauly J, Conolly S, et al. Background suppression with multiple inversion recovery nulling: applications to projective angiography. Magn Reson Med 1997;37:898-905.

32 Merino JG, Luby M, Benson RT, et al. Predictors of acute stroke mimics in 8187 patients referred to a stroke service. J Stroke Cerebrovasc Dis 2013;22:e397-403.

33 Dawson A, Cloud GC, Pereira AC, et al. Stroke mimic diagnoses presenting to a hyperacute stroke unit. Clin Med 2016;16:423-6.

34 Gargalas S, Weeks R, Khan-Bourne N, et al. Incidence and outcome of functional stroke mimics admitted to a hyperacute stroke unit. $J$ Neurol Neurosurg Psychiatry 2017:88:2-6.

35 Nagesh C, Kumar S, Menon R, et al. The imaging of localization related symptomatic epilepsies: the value of arterial spin labelling based magnetic resonance perfusion. Korean J Radiol 2018;19:965-77

36 Grams RW, Kidwell CS, Doshi $\mathrm{AH}$, et al. Tissue-Negative transient ischemic attack: is there a role for perfusion MRI? AJR Am J Roentgenol 2016;207:157-62.

37 Mlynash M, Olivot J-M, Tong DC, et al. Yield of combined perfusion and diffusion MR imaging in hemispheric TIA. Neurology 2009;72:1127-33.

38 Kleinman JT, Zaharchuk G, Mlynash M, et al. Automated perfusion imaging for the evaluation of transient ischemic attack. Stroke 2012;43:1556-60.

39 Pacheco FT, Allegro BB, Padilha IG, et al. Added value of arterial spin-labeling (ASL) and susceptibility weighted-images (SWI) in detecting a mild symptomatic intracranial arteriovenous fistula. Arq Neuropsiquiatr 2018;76:568

40 Noguchi T, Yakushiji Y, Nishihara M, et al. Arterial spin-labeling in central nervous system infection. Magn Reson Med Sci 2016;15:386-94.

41 Thamm T, Zweynert S, Piper SK, et al. Diagnostic and prognostic benefit of arterial spin labeling in subacute stroke. Brain Behav 2019;9:e01271.

42 Nael K, Meshksar A, Liebeskind DS, et al. Quantitative analysis of hypoperfusion in acute stroke: arterial spin labeling versus dynamic susceptibility contrast. Stroke 2013;44:3090-6.

43 Nael K, Meshksar A, Liebeskind DS, et al. Periprocedural arterial spin labeling and dynamic susceptibility contrast perfusion in detection of cerebral blood flow in patients with acute ischemic syndrome. Stroke 2013;44:664-70.

44 Okazaki S, Yamagami H, Yoshimoto T, et al. Cerebral hyperperfusion on arterial spin labeling MRI after reperfusion therapy is related to hemorrhagic transformation. J Cereb Blood Flow Metab 2017;37:3087-90.

45 Viallon M, Altrichter S, Pereira VM, et al. Combined use of pulsed arterial spin-labeling and susceptibility-weighted imaging in stroke at 3T. Eur Neurol 2010;64:286-96.

46 Crisi G, Filice S, Scoditti U. Arterial spin labeling MRI to measure cerebral blood flow in untreated ischemic stroke. $J$ Neuroimaging 2019;29:193-7. 